\title{
Research on the Integration of Blockchain and Ecological Agricultural Product Supply Chain -Based on the Survey in Southern Jiangxi
}

\author{
Wei Zhang ${ }^{1, *} \mathrm{Hui} \mathrm{Li}^{2}$ Wenzhe Luo ${ }^{1}$ \\ ${ }^{1}$ School of Economics and Trade, Nanchang Institute of Technology, Nanchang, China \\ ${ }^{2}$ Financial Department, Nanchang Institute of Technology, Nanchang, China \\ *Corresponding author. Email: 314527051@qq.com
}

\begin{abstract}
The characteristics of blockchain technology, such as decentralization, distributed storage, openness and transparency, can effectively solve the main problems in the supply chain of ecological agricultural products in southern Jiangxi province of China. With the "Internet of Things +", "big data +" and "blockchain+" era background, the blockchain and ecological agricultural products supply chain pattern should be promoted. Through the investigation in southern Jiangxi province, it can be found that the organic integration of blockchain and ecological agricultural products supply chain has practical guiding value for the improvement of ecological agricultural products supply chain in southern Jiangxi province. At the same time, it provides a reference for the reform of ecological agricultural products supply chain in China, and provides more possibilities for encouraging the development of ecological agriculture in China.
\end{abstract}

Keywords: Blockchain, Supply chain of agricultural products, Organic integration.

\section{INTRODUCTION}

At present, the standardization degree of ecological agricultural products in China is still relatively low, and the efficiency of supply chain is low, which is also the most important factor restricting the development of ecological agriculture. How to improve the problems existing in the supply chain of ecological agricultural products and promote the high-quality development of China's agriculture is of great significance to the development of China's agriculture [1]. The Rural Revitalization Strategic Plan (2018-2022) issued by the Communist Party of China Central Committee and The State Council states that "new agricultural and rural industries and new forms of business should be cultivated, new carriers and new models should be created for the integrated development of rural industries, and crossborder allocation of factors and organic integration of industries should be promoted". It can be seen that the improvement of ecological agricultural product supply chain in China still needs to rely on science and technology and innovation to create an efficient agricultural product supply chain model.

In recent years, blockchain, as a new force in Internet technology, has made rapid development. Blockchain can provide a transparent data and sharing environment, which is essentially a distributed shared ledger system that can be infinitely increased, encrypted, sequential, and decentralized [2]. Blockchain uses distributed ledgers that are not controlled by intermediaries such as banks and governments to keep transaction records. It has been successfully applied to many fields such as virtual currency and Internet finance, and successfully solved the problems of opaque information, low trust and high transaction costs in the transaction process [3]. At the same time, blockchain can realize the characteristics of immutable data and complete traceability, and can provide technical support for the traceability system of agricultural products [4]. In view of the current low efficiency of China's ecological agricultural product supply chain, blockchain has a good application prospect in this field with its excellent technical advantages.

Based on the field investigation of southern Jiangxi ecological agricultural products supply chain, this paper analyzes the present problems, and puts forward the organic integration of blockchain and ecological agricultural product supply chain. This conforms to the requirements of the rural vitalization strategy in China and has practical guiding value for the improvement of 
the supply chain of ecological agricultural products in southern Jiangxi.

\section{PROBLEMS EXISTING IN THE SUPPLY CHAIN OF ECOLOGICAL AGRICULTURAL PRODUCTS IN SOUTHERN JIANGXI}

\subsection{Poor Circulation of Information of Agricultural Products}

The ecological agricultural products in the South of Jiangxi Province are of various varieties and scattered distribution, and the circulation information is blocked. The circulation of agricultural products information among farmers, distributors, consumers and relevant government departments is not smooth. Under the existing operation mode of agricultural product supply chain, users at different stages participate in each link in different roles, and the information grasped by all parties has typical asymmetry. In order to safeguard their own interests, information barriers are serious, leading to the disconnection between production, supply and marketing. In southern Jiangxi province ecological agricultural products circulation mode is multistage retail mode, the supply chain participants mainly use the Internet of Things to realize the information collection, transmission, processing, processing and other business, but because of the Internet of Things system construction in different participants within the system, points belong to different platform, business are relatively independent. So the model could not quickly and efficiently to complete the information exchange. It is very difficult to realize the sharing and display of the whole process.

\subsection{Low Degree of Specialization and Standardization in the Agricultural Supply Chain}

The supply chain of ecological agricultural products in south Jiangxi province covers all links of "productionsupply-marketing", including production, logistics and sales, consumer consumption and other activities, involving a wide range of content, and the supply chain shows the characteristics of low degree of specialization and standardization. The ecological agricultural products in south Jiangxi mainly include navel orange, tea, chicken, etc., and the main body is generally individual farmers and small-scale cooperatives, and the scale is small. The level of specialization of such farmers is low, and they cannot make timely and effective use of new technologies and equipment to improve the production efficiency, quality of agricultural products and logistics speed, etc. In addition, the government departments pay relatively weak attention to agricultural business entities such as cooperatives and individual farmers. At present, the standardized and specialized agricultural products production in southern Jiangxi province only occupies a small market share. The ecological agricultural products still present the situation of "big market, small production" in the circulation field, and the supply chain efficiency is low.

\section{INTEGRATION OF BLOCKCHAIN AND ECOLOGICAL AGRICULTURAL SUPPLY CHAIN}

\subsection{The Characteristics of Integration of Blockchain and Ecological Agricultural Product Supply Chain}

Blockchain is an open ledger that uses computer programs to record all transaction information on the whole network. It is a decentralized and trust-free new data architecture. The organic integration of blockchain and ecological agricultural products has the following characteristics.

First, decentralization. Blockchain technology does not rely on third-party management or hardware facilities, there is no central control, through distributed accounting and storage, each node to achieve self-verification, delivery and management of information.

Second, distributed storage, open and transparent The essence of a blockchain is a mutually-validated public ledger system, and what it does is it records all the transactions that take place across all the accounts. The foundation of the blockchain technology is open source. In addition to the private information of the parties to the transaction being encrypted, the data of the blockchain is open to everyone.

Third, consensus mechanism. Based on consensusbased norms and protocols, in the organic integration of blockchain and ecological agricultural supply chain, the whole system does not rely on a third party, and all nodes can automatically and securely verify and exchange data within the system, without any human intervention, forming a consensus mechanism.

\subsection{The Feasibility of Integration of Blockchain and Ecological Agricultural Supply Chain}

\subsubsection{Policy Support}

The integration of blockchain and ecological agricultural supply chain has been supported by relevant policies in recent years. The Strategic Plan for Rural Revitalization (2018-2022) issued by the CPC Central Committee and The State Council states that: We need to seize the opportunities presented by important changes in the pattern of urban and rural development, foster new industries and new forms of business in agriculture and rural areas, create new carriers and new models for 
integrated development of rural industries, promote cross-border allocation of factors and organic integration of industries, and enable rural primary, secondary and tertiary industries to upgrade, add value and benefit simultaneously from integrated development. China's "13th Five-Year" Information Planning also mentioned that the" blockchain + "as an important means to solve the business problems of the industry in the future, by strengthening the blockchain technology scene application test, accelerate the integration of the blockchain technology and the industry, as soon as possible to form a mature solution.

In addition, jiangxi government has issued a number of policies to encourage blockchain technology, cultivate jiangxi blockchain industry cluster, and promote the development of Jiangxi blockchain industry. In January 2018, Jiangxi Provincial People's Government issued the Implementation Rules for the Construction of Ganjiang New Area Green Finance Reform and Innovation Pilot Zone, which mentioned the promotion and application of big data, cloud computing, block chain and other financial technologies to serve the development of green finance.

\subsubsection{Technical Support}

Blockchain technology has made many breakthroughs and developed rapidly in recent years. From the perspective of its own technology, blockchain is constantly evolving and enhancing, such as scalability, security, including some other processing power and other performance is constantly evolving. From the perspective of openness, it is moving from a closed state in the past to a cross-chain open state, as well as the combination of hardware and software to improve performance. From the perspective of the convergence of blockchain and other technologies, cases of the combination of blockchain and ICT are emerging, such as the development of blockchain and cloud computing, the Internet of Things, and big data.

\section{THE PATH OF BLOCKCHAIN TO OPTIMIZE THE ECOLOGICAL AGRICULTURAL SUPPLY CHAIN}

\subsection{Distributed Ledger to Realize Information Sharing of Ecological Agricultural Products Supply Chain}

A distributed ledger is a database that is shared, replicated, and synchronized among users. The transaction records are made by multiple nodes in different places, and each node records a complete ledger. Distributed ledger breaks the traditional mode of information transmission, so as to realize the information sharing of ecological agricultural supply chain.
The integration of blockchain distributed ledger technology and ecological agricultural products supply chain firstly connects all information islands into a whole through blockchain and Internet of Things technology platform, so that the information of ecological agricultural products can be smoothly circulated. Then, the transaction information of each node in the network is written into the ledger after passing the consensus verification, and it cannot be arbitrarily changed, and it does not need to be dominated by the core enterprises in the supply chain. Each nodal enterprise in the supply chain can get a copy, and each copy records all the transaction records of the whole supply chain, including the complete account of the information on the chain, and realizes the transmission of ecological agricultural products information.

\subsection{P2P Protocol to Improve the Efficiency of Ecological Agricultural Supply Chain Management}

P2P protocol, also known as point-to-point protocol, is both a technology and an idea. The decentralization of blockchain technology is to change the current sitecentric state of the Internet, so that the power is really in the hands of users, so as to improve the efficiency of supply chain management.

In the circulation of ecological agricultural products in southern Jiangxi province in the traditional supply chain mode, the information resources are mainly concentrated in the core enterprises, the circulation links are complicated, and the management efficiency is low. By applying decentralized technology to the ecological agricultural supply chain, information resources from the traditional core enterprises to the agricultural supply chain network nodes can directly exchange with each other freely, the corresponding information transmission efficiency will also be improved, and the efficiency of supply chain management will also be improved. For example, in the traditional agricultural product supply chain network, if wholesalers want to know the production and planting situation of upstream farmers, they can only know through the core enterprises. In this way, wholesalers are in a passive state and the communication efficiency is low. Moreover, core enterprises do not necessarily show all the information to wholesalers, and data sharing channels are not transparent. When the decentralization technology is applied, wholesalers can directly connect with farmers on the "chain", simplify the supply chain link, and ensure the openness and transparency of the "chain" data, make the information transmission more efficient, and optimize the efficiency of ecological agricultural supply chain management. 


\subsection{The Consensus Mechanism Can Enhance the Authenticity and Reliability of the Traceability Information of Agricultural Products Supply Chain}

The consensus mechanism of the distributed nodes in the blockchain system enables each region to form a "chain" that can store data forever and can continue indefinitely. The immutable time stamp effectively maintains the authenticity of information data, while the immutable data bar marks the transaction information on the whole network with a unique brand, which provides a new idea for information security and data tracking.

The information traceability system based on the blockchain technology architecture mainly functions at the nodes of the supply chain such as farmers, enterprises, wholesalers, retailers, consumers and government regulatory authorities. Taking the circulation mode dominated by leading enterprises in southern Jiangxi as an example, producers distribute sensors in the production base of ecological agricultural products, collect and transmit the information in the production process in real time, such as the temperature, humidity and ultraviolet intensity of the base, and then record the node database directly on the "chain". Companies with real and reliable information can use comprehensive information to better plan production and achieve a virtuous cycle. Farmers use unique FRID tracing source code or QR code to mark the produced agricultural products through permissions. After other nodes obtain corresponding permissions, information data synchronization can be realized through information collection and management. Government regulators can supervise the whole process at any time through their authority. Similarly, consumers can also scan RFID electronic tags or QR code to query information about all activities of the agricultural products from production to sales. In addition, they can also send complaints, comments and other feedback to the chain just like the ecommerce platform, so as to strengthen the supervision of the quality of ecological agricultural products and enhance the authenticity of the traceability information of the supply chain.

\section{CONCLUSIONS}

It is a solid foundation to optimize the supply chain system of agricultural products to continuously promote the construction of agricultural intelligent infrastructure, build modern agricultural intelligent information service platform and establish ecological agricultural products traceability system. The firmness of the supply chain system is closely related to the effective circulation of information, the fair trading of the platform and the cooperation of the participating enterprises. The application of blockchain will undoubtedly be greatly optimized for the above points. Based on the investigation of southern Jiangxi Province, this paper systematically sorted out the problems existing in the ecological agricultural products supply chain in southern Jiangxi by combining theory with practice, and innovatively proposed the organic integration of blockchain and ecological agricultural products supply chain.The integration of blockchain and ecological agricultural products supply chain will greatly improve the current state of ecological agricultural products in southern Jiangxi province.

But objectively speaking, there is still a long way to go to fully apply blockchain technology in the ecological agricultural supply chain system. Moreover, as a new underlying technology, blockchain is worthy of further analysis and research on how to integrate the original business system, and how to further combine with $5 \mathrm{G}$ technology and cloud computing.

\section{REFERENCES}

[1] Chen Fangguo, Wu Yunfei, Wang Shuaifei, Zhu Xidong. Research on supply chain model of Agricultural products in China. Southern Agricultural Machinery, 2019, 50(01):14-15. (In Chinese)

[2] Gai, Kim-K wang Raymond Choo. Controllable and trust worthy block chain-based cloud data management. Future Generation Computer Systems, 2019 (5): 527-535.

[3] Hokey Min. Enhancement of supply chain resilience by enhancing supply chain value. Journal of Business Horizons, 2018, 25 (3): 462-488

[4] Zhang Xiaheng. Optimization of supply chain management model based on blockchain. China Circulation Economy, 2018, 32(08): 42-50. (In Chinese)

[5] Fu Hao, Zhao Cuiping, Cheng Chuanxing. Blockchain embedding, constraint breaking and agricultural industry chain governance. Issues in Agricultural Economy, 2019, (12):108-117. (In Chinese)

[6] Alex Hughes, Andrew Park, Jan Kietzmann, Chris Archer-Brown. Beyond Bitcoin: Blockchain and Distributed Ledger Technologies for Firms. Business Horizons, 2019(2), 82. 